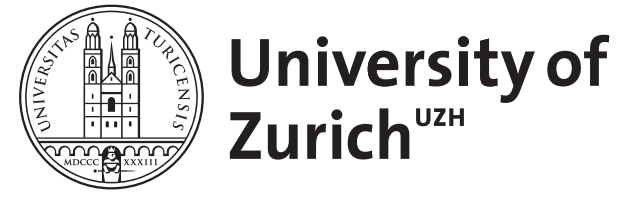

Zurich Open Repository and Archive

University of Zurich

University Library

Strickhofstrasse 39

CH-8057 Zurich

www.zora.uzh.ch

Year: 1989

\title{
Die Anschauungsformen und das Schematismuskapitel
}

Mudroch, Vilem

DOI: https://doi.org/10.1515/kant.1989.80.1-4.405

Posted at the Zurich Open Repository and Archive, University of Zurich

ZORA URL: https://doi.org/10.5167/uzh-80502

Journal Article

Published Version

Originally published at:

Mudroch, Vilem (1989). Die Anschauungsformen und das Schematismuskapitel. Kant-Studien, 80:405415.

DOI: https://doi.org/10.1515/kant.1989.80.1-4.405 


\title{
Die Anschauungsformen und das Schematismuskapitel
}

\author{
von Vilem Mudroch, Baden/Schweiz
}

Ein wesentlicher Unterschied zwischen der 1. und 2. Auflage der Kr.d.r.V. besteht in den unterschiedlichen Aufgaben, die jeweils dem Raum und der Zeit zugeordnet werden. Während in der 1. Auflage die Zeit eine auffällig dominierende Stellung einnimmt, ändert sich das Verhältnis der zwei Formen der Sinnlichkeit in der 2. Auflage zugunsten des Raumes. Eine der wenigen Stellen, die von dieser Wende unberührt bleiben, ist das Schematismuskapitel; eine Tatsache, die auf zwei Weisen erklärbar ist. Einerseits ist es denkbar, daß es sich einfach um ein Versäumnis handelt: hätte Kant mehr Zeit zur Überarbeitung der Kr.d.r.V. gehabt, hätte er im Schematismuskapitel auch dem Raum eine wesentliche Rolle zugeschrieben. Anderseits ist es aber möglich, daß Kant das Schematismuskapitel absichtlich unverändert ließ, weil es nämlich schon in der ursprünglichen Fassung den Anforderungen der kritischen Philosophie bestens genügte. In der vorliegenden Arbeit soll gezeigt werden, daß entgegen der Meinung vieler Kommentatoren ${ }^{1}$ eher die zweite Möglichkeit ${ }^{2}$ wahrscheinlich ist - dies vor allem aus Gründen der Architektonik.

Zum Ausgang werden einige mögliche, m. E. aber nicht hinreichende Argumente für und gegen den Einbezug des Raumes ins Schematismuskapitel untersucht (Abschnitt I). Weiter soll 1. auf den systematischen Ort der Schemata innerhalb des Begriffssystems der theoretischen Philosophie Kants und 2. auf die Rolle, die dort Zeit und Raum spielen, hingewiesen werden (Abschnitt II). Im Abschnitt III versuchen wir zu zeigen, daß die Aufmerksamkeit, die Kant in der 2. Auflage dem Raum widmet, mit dem Materiebegriff zusammenhängt. Dies ist für das Schematismuskapitel von entscheidender Bedeutung.

1 Z. B. H.J. Paton, Kant's Metaphysic of Experience (London: George Allen \& Unwin, Ltd. 1951), p.28n., 43n. N. Kemp Smith, A Commentary to Kant's Critique of Pure Reason (Atlantic Highlands: Humanities Press 1962), p.341. G.E. Franzwa, Space and Schematism, KantStudien, Bd.69, 1978, p. 149-159.

? Diese Alternative scheint wenige Anhänger gefunden zu haben. Unterstützt wird sie - mehr oder weniger explizit - von H. Cohen, Kants Theorie der Erfabrung (Berlin: Bruno Cassirer 19183); N. Rotenstreich, Kant's Schematism in Its Context, Dialectica X, 1956, p. 9-30; J. Simon, Sprache und Raum. Philosophische Untersuchungen zum Verhältnis zwischen Wabrbeit und Bestimmtbeit von Sätzen (Berlin: Walter de Gruyter 1969). 
Es gibt mindestens fünf denkbare Argumente, mit denen man die Forderung nach dem Einbezug des Raumes ins Schematismuskapitel unterstützen könnte.

(1) Ausgehend von der allgemeinen Aufwertung des Raumes in der zweiten Auflage der Kr.d.r. V. könnte man - wie eingangs erwähnt - die Meinung vertreten, daß auch der Raum in das Schematismuskapitel einbezogen werden müßte. Dieses Argument allein ist nicht hinreichend, da die Zeit in der 2. Auflage auch außerhalb des Schematismuskapitels, trotz Einschränkungen, weiterhin eine wichtige Rolle spielt. Die Abänderungen der 2. Auflage lassen keineswegs den Schluß zu, daß es sich hier nur um den Anfang einer Überarbeitung handelt, die noch fortgesetzt werden müßte. Es ist durchaus möglich, daß die ganze Entwicklung mit dem bereits Umgestalteten abgeschlossen ist.

(2) Zeit und Raum werden von Kant in der Transzendentalen Ästhetik in analogen Verfahren vorgestellt und weisen wichtige Ähnlichkeiten auf. Dies mag dazu veranlassen, die Parallelität der zwei Sinnlichkeitsformen für weite Teile der theoretischen Philosophie Kants geltend zu machen. Man könnte z. B. der Ansicht sein, daß das Vorhandensein zeitlicher Schemata mit der Angabe räumlicher Schemata aufzuwiegen sei. Man darf sich jedoch von solchen Überlegungen nicht irreführen lassen. Schon in der Transzendentalen Ästhetik gilt die Analogie höchstens für $\$ 2,3$ und $\$ 4,5$, erfährt aber wichtige Einschränkungen im $\$ 6$. In weiteren Teilen der Kr.d.r. V., vor allem aber in den $M . A$. und im Opus postumum werden die Unterschiede zwischen Zeit und Raum immer deutlicher.

Die nächsten drei Argumente für eine Abänderung des Schematismuskapitels sind gewichtiger. Jedes von ihnen würde - im Zusammenhang mit dem ersten Argument und der Tatsache, daß die reinen Verstandesbegriffe ursprünglich weder rein räumlich, noch rein zeitlich sind ${ }^{3}$ - die notwendigen und hinreichenden Möglichkeitsbedingungen der Angabe räumlicher Schemata liefern.

(3) Das dritte Argument stützt sich auf die Tatsache, daß die Naturwissenschaft nur unter Voraussetzung des Raumes möglich ist und eine sich nur auf Zeit gründende "eigentlich so genannte "Wissenschaft, z.B. die Psychologie, für Kant gar nicht in Frage kommt ${ }^{4}$. Man könnte daraus den Schluß ziehen, daß die räumlichen Schemata unentbehrlich und sogar von größerer Bedeutung als die zeitlichen sind. Wie wir in den Abschnitten II und III sehen werden, sind hier zwar die Prämissen richtig, nicht aber die Schlußfolgerung.

(4) Ähnliches kann man auch vom vierten Argument sagen, das davon ausgeht, daß die Schemata allein schwer zu verstehen sind und man zu ihrer vollständigen Interpretation die Grundsätze herbeiziehen muß, in denen Kant neben der Zeit auch den Raum berücksichtigt ${ }^{5}$. So ist z. B. beim Schema der Substanz, das Kant als „die Beharrlichkeit

${ }^{3}$ Vgl. J.Simon, Sprache und Raum, p. 90.

${ }_{4}$ Dies wird schon in den Paralogismen angedeutet und in den M.A. (IV, 471) explizit festgelegt.

5 Dies ist die Ansicht von H.J. Paton, Kant's Metaphysic of Experience, p. 44-60, der die Schemata hauptsächlich in ihrem Zusammenhang mit den Grundsätzen versteht. 
des Realen in der Zeit" (A 143 / B 183) beschreibt, nicht klar, was das Reale sein soll6. Zudem wird Substanz später (in den $M . A$.) nur räumlich aufgefaßt, und ihr jede zeitliche Bedeutung aberkannt (IV, 503, 542). Wir werden dieses wie auch das nächste Argument im nächsten Abschnitt diskutieren.

(5) Das fünfte Argument betrifft die Anwendung von Zeitverhältnissen auf äußere Gegenstände. Es wurde behauptet, dies sei nur dann möglich, wenn der Raum im Schematismuskapitel berücksichtigt werde. Wird diese Bedingung nicht erfüllt, so könne man nicht einmal die Zeit unserer Vorstellungen von der Zeit äußerer Gegenstände unterscheiden?.

Betrachten wir drei mögliche, m.E. aber nicht stichhaltige Erklärungen für das Fehlen des Raumes im Schematismuskapitel.

(a) Das Argument, daß Kant das Schematismuskapitel wegen Zeitmangels nicht überarbeitete, verliert an Glaubwürdigkeit, wenn man bedenkt, daß das Schematismuskapitel das einzige Kapitel (bzw. Teil, Abteilung) zwischen der Vorrede und den Paralogismen ist, das in der 2. Auflage unverändert bleibt. Man könnte durchaus meinen, Kant hätte die Zeit - und bei seiner enormen Arbeitskapazität sicher auch den Willen und die Kraft - zur Überarbeitung des Schematismuskapitels gefunden, hätte er es für erforderlich gehalten, den Raum einzubeziehen.

(b) Auch nicht restlos überzeugend ist der Einwand, daß die Zeit im Schematismuskapitel deshalb verwendet werde, weil sie, im Gegensatz zum Raum, eine klare Ordnung zum Ausdruck bringe und die Unterscheidung zwischen einem Fortschritt und einem Rückschritt ermögliche ${ }^{8}$. Eine andere Auffassung, die aber ähnlich beantwortet werden kann, lautet wie folgt: Nur die Zeit enthält ein Mannigfaltiges a priori in der reinen Anschauung ganz allgemein, ohne nähere Bestimmung, einzig zufolge der Regel des Nacheinander und entspricht daher in ihrer Allgemeinheit der Vorstellung eines reinen Materiäls für die allgemeine, transzendentale Einheit durch den Verstand. Der Raum enthält hingegen schon als reiner eigene Verhältnisse, z. B. die Vorstellung verschiedener Richtungen in ihm, die der absoluten Einheit durch den transzendentalen Begriff nicht entsprechen'. Überzeugend sind diese Argumente deshalb nicht, weil die Annahme, daß das Nacheinandersein allein eine klare bzw. allgemeine Ordnung ermögliche, nicht bewiesen wird ${ }^{10}$. In der Tat hat jede der zwei Sinnlichkeitsformen einen Nach- und einen Vorteil: in der Zeit wird zwar das Nach- und nicht das Nebeneinander bestimmt, im Raum wird umgekehrt das Neben- nicht das Nacheinander festgelegt. Auch wenn das Nebeneinander kompliziertere Verhältnisse impliziert als das eindimensionale Nacheinander, besteht noch kein Anlaß zur Behauptung, der Raum schaffe keine klare Ordnung oder sei weniger allgemein.

6 Siehe G. Franzwa, Space and Schematism, p. 153.

7. Ibid., p. 152, 155.

${ }^{8}$ N. Rotenstreich, Kant's Schematism, p. 15.

9 J.Simon, Sprache und Raum, p. 91.

${ }_{10}$ Die Allgemeinheitsforderung - anders formuliert und bewiesen - spielt dennoch eine Schlüsselrolle. Vgl. Abschnitt III. 
(c) Hermann Cohen war der Ansicht, daß räumliche Schemata deshalb unnötig scien, weil räumliche Verhältnisse in zeitlichen schon mitenthalten sind. Er schrieb, daß .im Schema auch das Räumliche nur als nacheinander auftauchende, nicht als nebeneinanderstehende Mehrheit von Elementen entworfen [wird]. In der Regel des Triangels werden die Konstruktionsstücke gedacht, nicht wie sie verbunden sind, sondern wie sie zu verbinden seien" "Obwohl es richtig ist, daß dic Schemata kein Bild, sondern Anwcisungen zur Konstruktion angeben (vgl. Abschnitt II), könnte man gegen Cohen einwenden, daß die Konstruktion zwar tatsächlich nacheinander, aber unter Gebrauch räumlicher Elemente geschieht, die durch zeitliche Regeln ungenügend bestimmt werden. So ist z. B. die in der Mathematik zentrale Idee der Konstruktion eines Begriffs, d.h. die Darstellung der $\mathrm{ihm}$ korrespondierenden Anschauung a priori (A 713/B 741), sowohl für die Geometrie, wie auch für die Arithmetik vorwiegend räumlich zu verstehen ${ }^{12}$. Cohens Argument beweist eigentlich nur die Unentbehrlichkeit zeitlicher Schemata. Wir können für das Folgende gelten lassen, daß die räumlichen Schemata die zeitlichen nie ersetzen, sondern höchstens ergänzen könnten.

Ein richtiges Verständnis der Rolle der Zeit im Schematismuskapitel ist nur im Hinblick auf die Gesamtheit der Begriffe der Transzendentalen Analytik und der daran anknüpfenden Werke möglich. Diese Begriffe müssen als hierarchisch angeordnet verstanden werden. Die Kategorien bilden die erste Gruppe, die am allgemeinsten und formalsten ist. Weiter folgen die wegen des Einbezugs einer Anschauungsform etwas weniger formalen und allgemeinen Schemata, dann die sich auf eine Natur überhaupt beziehenden reinen Grundsätze des Verstandes, weiter die Prinzipien der M.A., die sich mit einer besonderen Natur beschäftigen, und schließlich die Übergangsbegriffe des Opus postumum, die am wenigsten allgemein, formal und abstrakt sind. $\mathrm{Zu}$ hermeneutischen Schwierigkeiten, wenn nicht gerade Verwirrungen, führt die Tatsache, daß Kant in seinen Werken beim Betrachten jeder dieser Gruppen von Begriffen die Frage ihres genauen Geltungsbereichs zunächst fast völlig außer acht läßt. Erst wenn er zur nächsten Gruppe kommt, wird die Bedeutung der vorherigen Gruppe festgelegt, da erst jetzt die Bedingungen ihre Anwendbarkeit deutlich werden.

Die reinen (d. h. nichtschematisierten) Kategorien verdanken ihre Allgemeinheit der Tatsache, daß sie an keine Art von Sinnlichkeit gebunden sind; explizit kommt dies aber erst im Schematismuskapitel zum Ausdruck ${ }^{13}$. Dort lesen wir, daß den Kategorien allein nur eine "logische Bedeutung der bloßen Einheit der Vorstellungen “ bleibt, „denen aber kein Gegenstand, mithin auch keine Bedeutung gegeben wird, die einen

"H. Cohen, Kants Theorie der Erfabrung, p. 493. Ähnlich J. Simon, Sprache und Raum, p. 91.

12 G. Martin, Arithmetik und Kombinatorik bei Kant (Berlin: Walter de Gruyter 1972), p. 113.

13 Allerdings klingt dies schon in der Transzendentalen Deduktion an, so z. B. in der 2. Auflage im $\$ 24$. 
Begriff vom Objekt abgeben könnte“. Die Schemata „restringieren die Kategorien“, d. h. sie schränken sie auf Bedingungen ein, „die außer dem Verstande liegen (nämlich in der Sinnlichkeit)“ (A 146/B 186). Mit anderen Worten gibt die Kategorie „[die] Regel (oder vielmehr [die] allgemeine ... Bedingung zu Regeln)", nicht aber „den Fall, worauf sie angewandt werden" soll. Diese Funktion erfüllen die Schemata, mittels deren die transzendentale Philosophie „die Bedingungen, unter welchen Gegenstände in Übereinstimmung mit jenen Begriffen gegeben werden können, in allgemeinen, aber hinreichenden Kennzeichen" darlegt (A 135-6/ B 174-5).

Dabei wird die Frage der Bedeutung der Schemata selber vorerst nicht thematisch; Kant läßt es zunächst offen, ob sie z. B. schon Erkenntnis darstellen - im Sinne einer Kombination von Begriff und Anschauung (A 51 / B 75). Denn im Rahmen des Schematismuskapitels ist nicht klar, ob die in den Schemata anzutreffende Anschauungsform der Zeit der Forderung Kants nach einer gegebenen Anschauung tatsächlich genügt ${ }^{14}$. Wie schon oben erwähnt, sind die Schemata ohne die Grundsätze kaum verständlich ${ }^{15}$. Die Grundsätze ihrerseits würden aber rätselhaft bleiben, wenn man nicht die Prinzipien der M.A. zur Hilfe ziehen könnte ${ }^{16}$. Damit wird auch das 4. Argument aus Abschnitt I beantwortet. Die Tatsache, daß die Schemata ohne die Grundsätze kaum verständlich sind, stellt keine Eigentümlichkeit dieser Begriffe dar; die relative Unverständlichkeit jeder Begriffsgruppe erweist sich als eine systembedingte Erscheinung.

Zu untersuchen ist nun die Rolle, die die Zeit und der Raum in der Hierarchie der Begriffe spielen. Wie schon gesagt, steht nicht fest, ob man die mit keiner eigenen anschaulichen Komponente ausgestatteten reinen Kategorien räumlich oder zeitlich auslegen soll. Das transzendentale Schema muß jedoch der Anforderung genügen, „einerseits mit der Kategorie, anderseits mit der Erscheinung in Gleichartigkeit" ${ }^{\circ} \mathrm{zu}$ stehen (A 138/B 177). Das transzendentale Schema muß also ein solches Merkmal aufweisen, das sowohl allgemein, wie auch in jeder empirischen Vorstellung des Mannigfaltigen enthalten ist (ibid.). Dieses Merkmal soll erstens bewirken, daß sich das transzendentale Schema nicht auf die einzelne Anschauung, sondern auf „die Einheit in der Bestimmung der Sinnlichkeit" richtet. Deshalb darf das Schema kein Bild sein, weil dieses nur singulär ist, sondern die „Vorstellung ... von einem allgemeinen Verfahren der Einbildungskraft, einem Begriff sein Bild zu verschaffen “ ist (A 140 / B 179-180). Zweitens muß das gesuchte Merkmal in jeder Erscheinung angetroffen werden, z.B. auch im empirischen Ich. Zudem muß es so gewählt sein, daß das Schema rein ist, sonst

14 Dies wird in der Allgemeinen Anmerkung zum System der Grundsätze in Frage gestellt. Siehe weiter unten.

15 Dies leuchtet ein, wenn man bedenkt, daß die Schemata die sinnlichen Bedingungen, unter welchen „reine Verstandesbegriffe allein gebraucht werden können“, darstellen, und die Grundsätze diejenigen Urteile sind, „welche aus reinen Verstandesbegriffen unter diesen Bedingungen a priori herfließen" (A 136/B 175). Das eigentliche Resultat der Transzendentalen Doktrin der Urteilskraft sind die Grundsätze. Dies erklärt auch,-warum das Schemarismuskapitel so verblüffend kurz ausfällt.

${ }^{16} \mathrm{~J}$. Vuillemin, La physique et métaphysique kantiennes (Paris: P.U.F. 1955). Das ganze Werk ist dem Ansatz verpflichtet, die Grundsätze anhand der Prinzipien $\operatorname{der} M$. A. zu erläutern. 
wäre nämlich die Verbindung zwischen der Kategorie und der Erscheinung nur empirisch". Kant meint, daß gerade die Zeit, ,als die formale Bedingung des Mannigfaltigen des inneren Sinnes, mithin der Verknüpfung aller Vorstellungen ${ }^{*}$ das gewünschte Merkmal darstellt (A 138/B 177).

Wir wollen jetzt untersuchen, ob diese Funktion nicht auch vom Raum erfüllt werden könnte, denn die Zeit weist cine wichtige Einschränkung auf. Die Zeit ist nämlich „nichts anders als die Form des inneren Sinnes, d.i. des Anschauens unserer selbst und unseres inneren Zustandes ... die Zeit kann keine Bestimmung äußerer Erscheinungen sein: sie gehört weder zu einer Gestalt, oder Lage" (A 33/B 49). Mit ihr allein können wir äußere Gegenstände kaum bestimmen; nur ihr Nacheinander ist mittels der Zeit festlcgbar, nicht aber ihre räumlichen Verhältnisse ${ }^{18}$.

Offensichtlich wäre es für Kant nicht sonderlich schwierig gewesen, räumliche Schemata aufzustellen. Dies sehen wir an den folgenden Beispielen. Als Schema der Größe nennt Kant die Zahl, , welche eine Vorstellung ist, die die sukzessive Addition von Einem zu Einem (gleichartigen) zusammenbefaßt" (A 142/B 182). Nun meint er zwar, daß die Zahl dadurch entsteht, "daß ich die Zeit selbst in der Apprehension der Anschauung erzeuge" (A 143/B 182). Doch liegt die Vorstellung sehr nahe, daß man die Zahl, außer auf die sukzessiv entwickelte Reihe in der Zeit, auch auf die ruhende Mannigfaltigkeit im Raume bezieht. Dies erscheint unproblematisch, wenn man bedenkt, daß nicht nur die Zeit, sondern auch der Raum gleichartig ist und die transzendentale Synthese, durch die wir Gegenstände erkennen, die gleichartigen Teile der Zeit und des Raumes synthetisiert. Außerdem kann man sich den Zeitablauf nur dann vorstellen, wenn man eine räumliche Anschauung zu Hilfe nimmt und eine Linie zieht (A 33 / B 50). So würde die Zahl, als Schema der Quantitär, sowohl für Zeitlänge als auch für Gestalt gelten.

Ähnlich könnte man die durch die drei Relationskategorien bestimmte Stellung eines Gegenstandes nicht nur zeitlich, wie dies Kant tut, sondern auch räumlich auffassen ${ }^{19}$. Ein solches Verfahren scheint sich im Falle des Schemas der Substanz aufzudrängen, weil hier von Beharrlichkeit des Realen in der Zeit die Rede sein wird. Es gibt gewichtige Gründe, „das Reale“ räumlich zu interpretieren. So erklärt Kant: „Der Zeit also, die selbst unwandelbar und bleibend ist, korrespondiert in der Erscheinung das Unwandelbare im Dasein, d.i. die Substanz, und bloß an ihr kann die Folge und das Zugleichsein der Erscheinungen der Zeit nach bestimmt werden" (A 143/B 183). Das "Unwandelbare im Dasein“ formt ein Pendant zur Zeit und ihren Bestimmungen; und man könnte zur Überzeugung gelangen, daß der Raum impliziert sein muß20.

17 H.J. Paton, Kant's Metaphysic of Experience, p. 28.

18 Es wäre übertrieben zu behaupten, $\mathrm{da} ß$ es ohne den Raum im Schematismuskapitel unmöglich wäre, Aussagen über jede Ordnung von äußeren Gegenständen zu machen. Vgl. G. Franzwa, Space and Schematism, p.152. In Wahrheit wären es nur die räumlichen Verhältnisse der äußeren Gegenstände, die unbestimmbar blieben.

19 H.J. Paton, Kant's Metaphysic of Experience, p. 52.

20 Hier hat man sich offensichtlich von der scheinbaren Parallelität zwischen Raum und Zeit irreführen lassen (vgl. I, Argument 2). 
Auch das Schema der Ursache und sogar die 2. Analogie könnten räumlich ausgelegt werden. Zwar gehören die Grundsätze des reinen Verstandes, streng genommen, nicht zu unserem Thema, denn dort wird, im Gegensatz zum Schematismuskapitel, der Raum berücksichtigt. Allerdings geschieht dies hauptsächlich in den Axiomen der Anschauung (A 162/B 203) und den Antizipationen der Wahrnehmung (A 173-4/ B 215-6), weniger in den Analogien, so daß wir diese für unsere Zwecke noch zusammen mit den Schematen betrachten dürfen. In der 2. Analogie geht es darum, eine subjektive von einer objektiven Zeitreihenfolge zu unterscheiden, was aufgrund der Ab- oder Anwesenheit einer Regel des Verstandes geschieht. Diese Regel wird sowohl im Schema der Ursache und der Kausalität (A 144/B 183), wie auch in der 2. Analogie zeitlich aufgefaßt: als notwendiges Nacheinander ${ }^{21}$. Eine räumliche Regel, gegründet auf dem notwendigen Nebeneinander, wäre durchaus denkbar. Allerdings würde hier nicht notwendiges unmittelbares Nebeneinander, sondern notwendiges Nebeneinander schlechthin als ein Kriterium dienen, denn Kant wollte auch Fernwirkung, d.h. die Gravitationskraft, zulassen.

Dennoch kann der Raum die im Schematismuskapitel der Zeit zugeschriebene Funktion nicht erfüllen. Nur die Zeit genügt der Allgemeinheitsforderung des Schematismus. Der Raum ist bloß auf spezifische Bereiche anwendbar, und die Aufwertung, die er in der zweiten Auflage der Kr.d.r.V. erfährt und die sich in den $M . A$. und im Opus postumum fortsetzt, verknüpft den Raum aufs engste mit dem empirischen Begriff der Materie.

Der Raum könnte es dem Schema nicht ermöglichen, „mit der Erscheinung in Gleichartigkeit“ zu stehen. „Die Zeit ist die formale Bedingung a priori aller Erscheinungen überhaupt. Der Raum als die reine Form aller äußeren Anschauung ist als Bedingung a priori bloß auf äußere Erscheinungen eingeschränkt ... Alle Vorstellungen ... sie mögen äußere Dinge zum Gegenstande haben oder nicht ... [gehören] zum inneren Zustande... Man kann hier von einer Vertretung des Raumes durch die Zeit sprechen $^{22}$ : Die Zeit ist „eine Bedingung a priori von aller Erscheinung überhaupt und zwar die unmittelbare Bedingung der inneren (unserer Seelen) und eben dadurch mittelbar auch der äußeren Erscheinungen“ (A 34 / B 50). In bezug auf den Schematismus heißt es dann, daß „das reine Bild aller Größen (quantorum) vor dem äußeren

21 Dies beantwortet das fünfte Argument aus Abschnitt I. Die Unterscheidung der Zeit unserer Vorstellungen von der Zeit äußerer Gegenstände wird nicht in bezug auf den Raum, sondern gerade durch die Ab-oder Anwesenheit einer Regel des Verstandes gemacht. Die von G. Franzwa (Space and Schematism, p. 153) eingeführte Unterscheidung zwischen einer subjektiven und einer phänomenalistischen Interpretation der Zeit ist irreführend, weil sie die von Kant selbst benutzte Unterscheidung einer subjektiven von einer objektiven Reihenfolge verdeckt.

22 J.Simon, Sprache und Raum, p.36-7. 
Sinne ... der Raum, aller Gegenstände der Sinne aber überhaupt die Zeit ${ }^{*}$ ist (A 142 ) B 182) ${ }^{23}$.

Dic Zeit ermöglicht nur schr dürftige Aussagen über äußere Gegenstānde und erlaubt kaum die Bestimmung der besonderen Natur. Aus diesem Grund nimmt Kant den Raum zunehmend zu Hilfe. Allerdings geht es bei der Aufwertung des Raumes hinsichtlich der Bchandlung besonderer Gegenstände nicht nur um den Raum selber, sondern vor allem um den mit Materie gefüllten Raum. Wie in diesem Abschnitt deutlich wird, fallen die Aussagen über den Raum als der reinen Form der Sinnlichkeit eher bescheiden aus, was damit zusammenhängt, daß der Raum ohne Materie kein Objekt der Wahrnehmung (IV, 559), oder - wie Kant im Opus postumum schreibt - kein Objekt der Erfahrung sein kann (XXII, 192). Dies soll einerseits die Unmöglichkeit sinnlicher Wahrnehmung des leeren Raumes unterstreichen, anderseits darauf hinweisen, daß man vom leeren Raum keine besonderen naturwissenschaftlichen Gesetze aufzustellen vermag ${ }^{24}$. So hätte aber die Aufwertung des Raumes in der 2. Auflage des Kr.d.r.V. für den Schematismus keine Bedeutung, weil der Materiebegriff empirisch ist (A 847-8 / B 875-6, IV, 470). Der Raum dürfte aus diesem Grund im Schematismuskapitel nicht vorkommen, denn er würde die Reinheit der Schemata verletzen.

Ein kurzer Vergleich der Begriffe "leerer Raum " und „leere Zeit" zeigt, warum der Raum und nicht die Zeit eine Aufwertung erfährt. Dafür ist nicht nur die Tatsache, daß die äußeren Verhältnisse der Gegenstände mit der eindimensionalen Zeit ungenügend bestimmt werden können, verantwortlich ${ }^{25}$. Wichtig ist auch, daß man die leere Zeit mit Vorstellungen füllen kann, von denen einige, wie z. B. Träume, gar nichts zur Erkenntnis beitragen können, andere, die mit einer Regel des Verstandes verbunden sind, zwar zur Erkenntnis führen, aber nur zu solcher von Objekten überhaupt. Besondere Erkenntnis ist hier deshalb nicht zu erwarten, weil diese Erkenntnis Materie als ihr Objekt hat. Materie aber kann nur den Raum und nicht die Zeit erfüllen. Darauf weist Kant schon in der Widerlegung des Idealismus hin, wo alle Zeitbestimmungen als von etwas Beharrlichen abhängig bezeichnet werden, und „dieses Beharrliche nur durch ein Ding außer mir und nicht durch die bloße Vorstellung eines Dinges außer mir möglich“ ist (B 275). Was das "Ding außer mir" ist, wird zunächst offen gelassen und erst in den M.A. weiter bestimmt.

Die mit der Einführung des Materiebegriffs verbundene Aufwertung des Raumes deutet sich also schon in der 2. Auflage der Kr.d.r.V. an, vor allem in der Allgemeinen Anmerkung zum System der Grundsätze. Dort stellt Kant fest, daß die objektive

${ }^{23}$ Die beiden letzten Stellen werden zwar überall zitiert (z. B. H.J. Paton, Kant's Metaphysic of Experience, p.28n., 78; N. Kemp Smith, Commentary, p.341; J.Simon, p. 91), m. E. aber ungenügend gewürdigt, da Kants architektonische Ziele nicht berücksichtigt werden.

24 Dies der dynamischen Hypothese zufolge (IV, 523-4), die keinen leeren Raum zuläßt. Diese Hypothese erweist sich aber in der Naturwissenschaft als sehr fruchtbar, denn sie dient der Newtonschen Physik als Grundlage. Die entgegengesetzte Hypothese, der Atomismus, macht vom leeren Raum Gebrauch, zeigte sich aber zu Kants Zeiten in der Naturwissenschaft als kaum verwendbar.

${ }^{25}$ Siehe IV, 471. 
Realität der Kategorien von der Anwesenheit äußerer Anschauungen abhängt und erläutert seine Behauptung am Beispiel der reinen Relationsbegriffe. So bedürfen wir, „um dem Begriffe der Substanz korrespondierend etwas Bebarrliches in der Anschauung zu geben (und dadurch die objektive Realität dieses Begriffs darzutun), ... eine Anschauung im Raume (der Materie) ... Um Veränderung, als die dem Begriffè der Kausalität korrespondierende Anschauung, darzustellen, müssen wir Bewegung als Veränderung im Raume zum Beispiele nehmen ..." (B 291) ${ }^{26}$ Die explizite Anerkennung der Verbindung des Raumes mit der Materie ist mit der nicht minder expliziten Feststellung der Grenze einer nur auf der inneren Form der Sinnlichkeit gründenden Erkenntnis verbunden. Für Kant ist es das Ziel der Allgemeinen Anmerkung, die Schranken der Möglichkeit einer Selbsterkenntnis „aus dem bloßen inneren Bewußtsein und der Bestimmung unserer Natur ohne Beihilfe äußerer empirischen Anschauungen“ anzuzeigen (B 293-4).

Die Allgemeine Anmerkung zum System der Grundsätze ist in der Kr.d.r. V. eher isoliert. Im Hauptstück System aller Grundsätze des reinen Verstandes wird zwar der Raum einige Male erwähnt, aber Materie und die mit ihr verbundenen Begriffe der Bewegung und Kraft werden systematisch vermieden. Dies führt zu einigen recht merkwürdigen Resultaten. So wird z. B. in den Antizipationen der Wahrnehmungen die Negation nicht - wie man erwarten würde - als eine negative Größe, sondern als Null aufgefaßt. Kant tut dies deshalb, weil er in den Antizipationen den von Materie unabhängigen Begriff des Lichtgrades, bei dem es keine negative Größe geben kann, als Paradigma nimmt und nicht etwa Bewegung oder Kraft, bei denen negative Größen möglich wären ${ }^{27}$.

So soll aber die Allgemeine Anmerkung nicht als ein Hinweis auf eine mögliche Korrektur der Kr.d.r.V., sondern eher als ein Programm für die Ausarbeitung der Prinzipien einer sich auf besondere Gegenstände beziehenden Erkenntnis verstanden werden. Die hier ansetzende Entwicklung wird vor allem in den M.A. und im Opus postumum weiter verfolgt. In den $M . A$. wird zwar die Rolle der reinen Anschauungsformen nicht bestritten, denn diese sind zur Mathematisierung unerläßlich (IV, 470), doch liegt das Hauptgewicht bei der stufenartigen Entfaltung des Materiebegriffs, bei der der Raum eine Schlüsselrolle spielt. Die meisten durch die vollständige Zergliederung des Materiebegrifs (IV, 472) entstandenen Prinzipien werden von Kant eher rāumlich als zeitlich aufgefaßt. Die folgenden vier Beispiele, in denen Raum und Materie aufs engste verknüpft werden, seien erwähnt ${ }^{28}$.

(1) Flüssigkeiten können entweder in Masse oder im Flusse wirken. Ein Beispiel des ersteren ist die Wirkung von Wasser auf eine Waagschale, des zweiteren die Wirkung

26 Ähnlich vollzieht Kant die Verknüpfung von Raum und Materie auch in den $M . A .(I V, 478)$, wo die „Form und Prinzipien der äußeren Anschauung“ im Zusammenhang mit der allgemeinen Körperlehre erwähnt werden.

27 Vgl. J. Vuillemin, La physique et métaphysique kantiennes, p. 132.

${ }^{28}$ Eine ausführliche Diskussion der Rolle des Raumes in Kants theoretischer Philosophie findet sich in V.Mudroch, Kants Theorie der physikalischen Gesetze, Kantstudien-Ergänzungshefte (Berlin: Walter de Gruyter 1987), Kap. 2. 
eines Mühlbachs auf die Schaufel eines Wasserrads. Masse wird demnach als ein Nebenund nicht als ein Nacheinander verstanden; auch Gleichzeitigkeit wird räumlich, als ein Nebeneinander begriffen ${ }^{29}$.

(2) Kant hat $m v$ als Formel der Erhaltung der Bewegungsquantität der Leibnizschen Formel $m v^{2}$ deshalb vorgezogen, weil nur die erstere die Quantität der Bewegung (und damit indirekt Quantität der Substanz) räumlich, d.h. vektorial angibt. Der Ausdruck $v^{2}$ kann nur einen positiven Werr haben und ermöglicht keine Richrungsangabe (positiv oder negativ) ${ }^{30}$.

(3) Das erste Gesetz der Mechanik, dem die 1. Analogie zugrunde liegt, lautet: „Bei allen Veränderungen der körperlichen Natur bleibt die Quantităt der Materie im Ganzen dieselbe, unvermehrt und unvermindert ${ }^{*}$ (IV, 541). In der anschließenden Anmerkung wird dieses Gesetz explizit auf räumliche Objekte eingeschränkt, da Substanz jetzt nur im Raume, als Gegenstand des äußeren Sinnes möglich ist.

(4) Das zweite Gesetz der Mechanik, das die zweite Analogie zugrunde legt und dem 1. Bewegungsgesetz Newtons entspricht, lautet: „Alle Veränderung der Materie hat eine äußere Ursache “ (IV, 543). In der 2. Analogie wurde vom „Äußeren“ noch nicht gesprochen, dort lag das Gewicht auf der Unterscheidung der objektiven von der subjektiven Reihenfolge der Wahrnehmungen.

Interessant sind auch Kants Äußerungen im Opus postumum, wo die in den M.A. angefangene Behandlung der besonderen Natur weitergeführt wird. Dort wird die nun zur Regel gewordene Verknüpfung des Raumes mit der Materie durch den Ausdruck "sensibler Raum ${ }^{\alpha 31}$ umschrieben, bei dem es sich nicht um die Form der Anschauung, sondern um einen durch Materie kontinuierlich erfüllten Raum handelt.

$\mathrm{Daß}$ Kant das Schematismuskapitel auch bei einer gründlicheren Überarbeitung der Kr.d.r.V. nicht abgeändert hätte, zeigt sich im Opus postumum daran, daß er immer noch bereit ist, die zeitlichen Schemata der Kr.d.r.V. zu gebrauchen, obwohl es für ihn sehr einfach gewesen wäre, in seinem letzten Werk die Lehre der Kr.d.r.V. zu korrigieren. So finden wir im Opus postumum eine Definition der Notwendigkeit, die dem Schema der Notwendigkeit in der Kr.d.r. V. sehr nahe kommt. In der Kr.d.r.V. heißt es: „Dasein eines Gegenstandes zu aller Zeit“ (A 145/B 184), im Opus postumum steht: „perpetuitas est necessitas phenomenon “ (XXII, 605).

Der Begriff des mit Materie erfüllten Raumes führt in Richtung besonderer Gegenstände und folglich auch empirischer Begriffe. Man könnte hier von einer Vermittlerrolle des Raumes zwischen reinen und empirischen Begriffen reden, wobei gerade die Ab- bzw. Anwesenheit des Materiebegriffs den Unterschied zwischen der Bestimmung des Raumes als transzendentaler Anschauungsform und dem Raum als Schema empirischer Begriffe erklärt ${ }^{32}$.

29 IV, 540; vgl. J. Vuillemin, p. 263.

so IV, 539; J. Vuillemin, p. 261.

31 Vor allem in der sogenannten transzendentalen Deduktion in X. und XI. Konvolut, z. B. XXI, 216; XXI, 228.

32 Siehe J. Simon, Sprache und Raum, p. 91 . Simon sieht hier eine Lücke, die aber m. E. durch den Materiebegriff gefüllt wird. 


\section{Schlußbemerkungen}

Die vorliegende Arbeit, die zu zeigen versucht, daß es sich beim Weglassen des Raumes im Schematismuskapitel um kein Versehen oder Versäumnis seitens Kants handelt, stellt die in der 2. Auflage vollzogene Aufwertung des Raumes in ein bestimmtes Licht. Nach unserer Interpretation wendet sich Kant dem Raum nicht deshalb zu, weil etwa der Raum weniger idealistische Momente impliziert, von denen sich Kant veranlaßt durch eine Fehlinterpretation eines Rezensenten der 1. Auflage der Kr.d.r. V. - bekanntlich distanzieren wollte. Auch geht es ihm nicht primär darum, mittels des Raumes die objektive Gültigkeit der reinen Begriffe des Verstandes zu sichern. Obwohl solche Überlegungen auch mitgespielt haben dürften, ging es Kant in erster Linie darum, eine Verbindung zwischen der Transzendentalen Analytik und der Philosophie der besonderen Natur herzustellen. In der 1. Auflage begnügte sich Kant damit, die allgemeinen Begriffe der Transzendentalphilosophie festzulegen, in der 2. Auflage sah er sich durch seine Arbeit an den $M$. A. gezwungen, einige Hinweise auf die Nützlichkeit und Grenzen dieser Begriffe angesichts der jetzt präzisierten Formulierung seiner Theorie der Naturwissenschaft zu geben. An denjenigen Stellen der 2. Auflage, an denen der Raum betont wird, deutet Kant an, daß ihn weit mehr als nur die logische Bedeutung der reinen Begriffe des Verstandes oder die Objekte einer Natur überhaupt interessiere. Er will sich nämlich mit neuen Geltungsbereichen auseinandersetzen. Und die neu aktuelle Frage ist nicht mehr nur nach der Bedeutung der reinen Begriffe schlechthin, sondern nach ihrer Bedeutung für die Erkenntnis der besonderen Natur.

Aber auch wenn Kant durch seine Arbeit an den $M$.A. die Wichtigkeit des Raumes und die Grenzen der Zeit deutlicher erkannte, heißt dies nicht, daß er seine Ausführungen in der 1. Auflage völlig aufgeben wollte. Diese stellen auch weiterhin den Grundstein seiner Philosophie dar und behalten dadurch ihren Wert. Kant wollte sein System mit den obersten Prinzipien anfangen und somit das erkenntnistheoretische Problem in seiner ganzen Allgemeinheit zum Ausdruck bringen. $\mathrm{Da}$ aber der Schematismus zu diesem hoch-abstrakten und allgemeinen Teil gehört, bleibt er von den Veränderungen der 2. Auflage ausgeschlossen. Darin liegt auch die Antwort auf Argument (3) aus Abschnitt I. Die Naturwissenschaft ist tatsächlich nur unter Voraussetzung des Raumes und des damit verbundenen Materiebegriffs möglich, aber das Schematismuskapitel ist eben nicht im Hinblick auf die spezifische Problemstellung der Philosophie der besonderen Natur konzipiert und ist mit der reinen Anschauungsform der Zeit bestens bedient.

Das Unbehagen, das der Nichteinbezug des Raumes in das Schematismuskapitel in der Literatur bewirkte, hat einen Grund. Die nur zeitlich konzipierten Schemata leisten nämlich sehr wenig. Berücksichtigt man aber auch architektonische Gesichtspunkte, wird Kants Ausschluß des Raumes aus dem Schematismuskapitel verständlich. 